\title{
Actualización y adaptación a la docencia híbrida de la asignatura Documentación y Metodología Científica del Grado en Farmacia
}

Rut Lucas-Domínguez ${ }^{\mathrm{a}}$, Antonio Vidal-Infer ${ }^{\mathrm{b}}$, Carmel Ferragud ${ }^{\mathrm{c}}$, Juan Carlos Valderrama-Zurián ${ }^{\mathrm{d}}$, Mar Cuenca Lorente ${ }^{\mathrm{e}}$

${ }^{a}$ Dpto. Historia de la Ciencia y Documentación. Universitat de València (Rut.Lucas@uv.es), ${ }^{b}$ Dpto. Historia de la Ciencia y Documentación. Universitat de València (Antonio.Vidal-Infer@uv.es). ${ }^{c}$ Dpto. Historia de la Ciencia y Documentación. Universitat de València (Carmel.Ferragud@uv.es), ${ }^{\mathrm{d} D p t o .}$ Historia de la Ciencia y Documentación. Universitat de València (Juan.Valderrama@uv.es), ${ }^{e}$ Dpto. Historia de la Ciencia y Documentación. Universitat de València (Maria.Mar.Cuenca@uv.es)

\section{\$EWWFW}

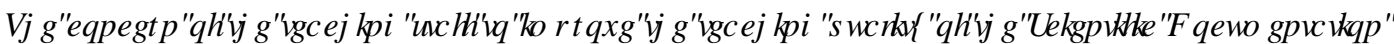

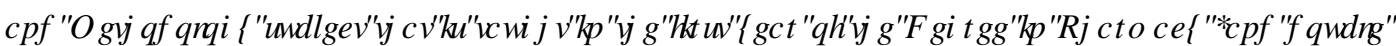

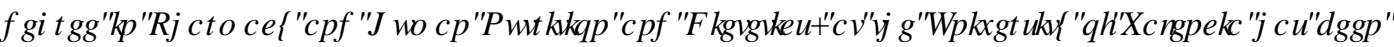

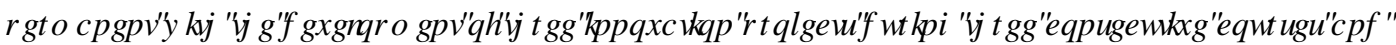

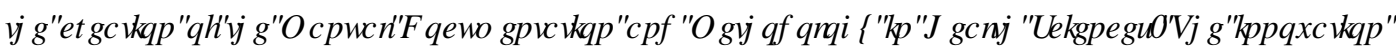

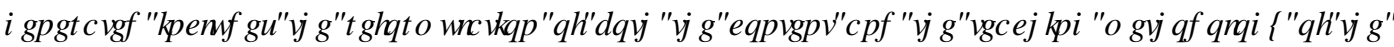

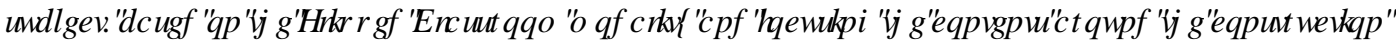

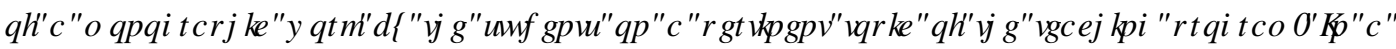

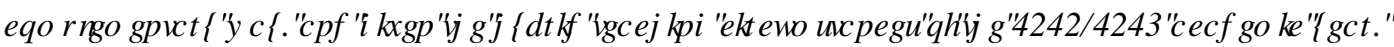

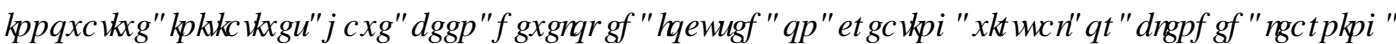

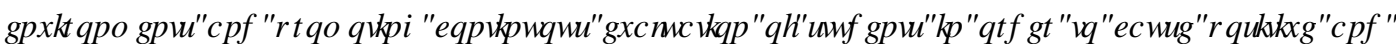

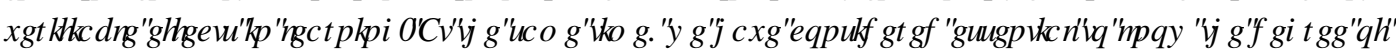

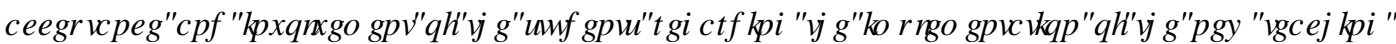
$P R G D O M H D$

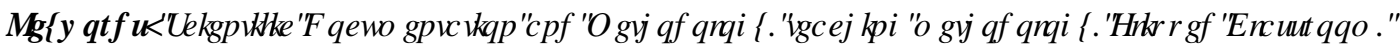

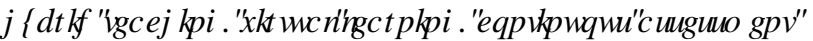

\section{HXP HQ}

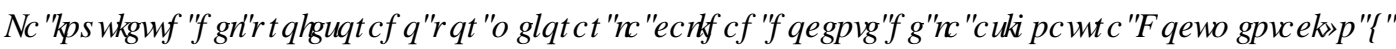

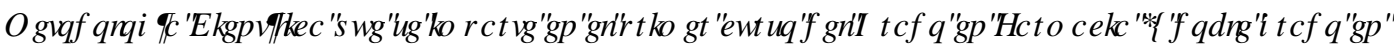

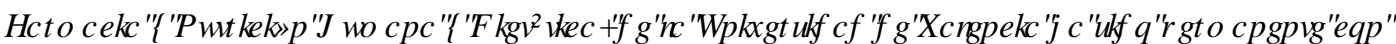

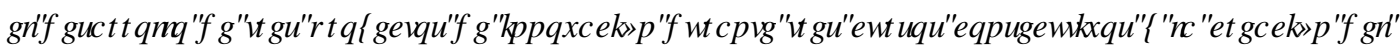

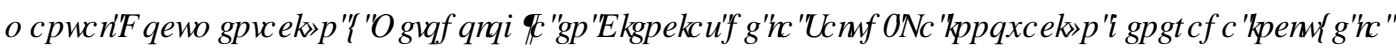

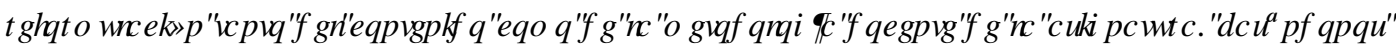

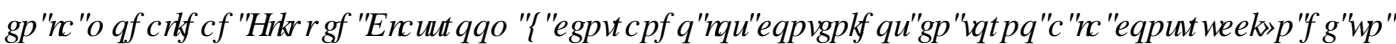

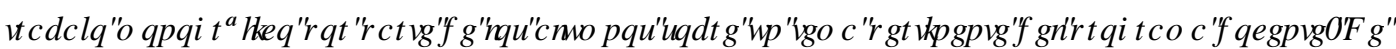

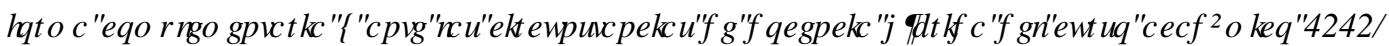

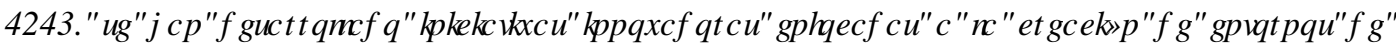

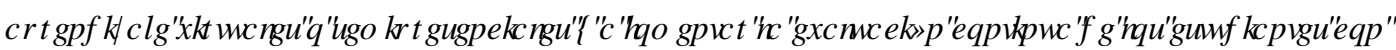

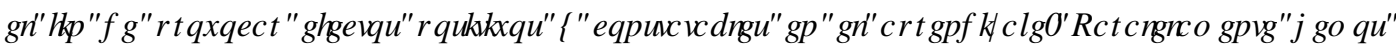

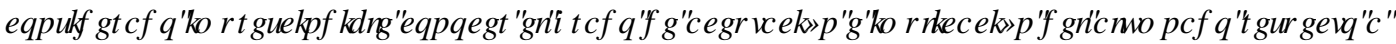

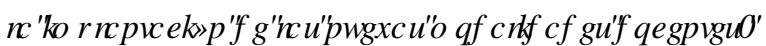




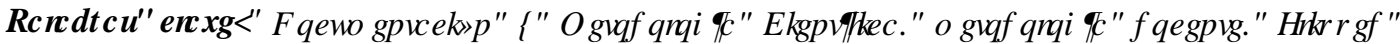

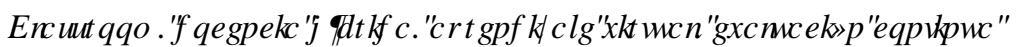

\section{Introducción}

Uno de los desafíos a los que el profesorado de la asignatura Documentación y Metodología Científica nos hemos enfrentado durante los últimos diez años es su ubicación dentro del plan de estudios de la titulación de Farmacia de la Universitat de València (UV) (ANECA, 2004). Con la creación del Espacio Europeo de Educación Superior (Declaración de Bolonia, 2009) esta asignatura se incorporó en el primer cuatrimestre del primer curso del Grado en Farmacia (y Doble Grado en Farmacia y Nutrición Humana y Dietética), donde los y las estudiantes aún están aterrizando en el mundo universitario y no tienen el bagaje más adecuado para asimilar los contenidos que impartimos. Sus conocimientos sobre la ciencia, la medicina y la farmacia son superficiales y sesgados, y gran parte de nuestro alumnado no cuenta con el interés suficiente para indagar en estas cuestiones. Por esta razón, la metodología docente tradicional basada en sesiones magistrales, acompañadas de unas clases prácticas, son insuficientes para conseguir los objetivos que nos proponemos.

Cabe decir que en el Grado en Farmacia existen cinco grupos. Tres de ellos tienen horario por las mañanas, en concreto el AR (inglés), B (castellano) y C (valenciano), y dos por la tarde, el D (castellano) y E (valenciano/castellano, según demanda). El alumnado perteneciente al doble grado está integrado en los grupos de Farmacia en esta asignatura. Normalmente, los grupos AR y E suelen ser bastante menos numerosos, en torno a los 40 estudiantes, frente a los 60 que suelen componer los grupos B, C y D. Es importante tener en cuenta este contingente para entender las dificultades para emprender metodologías participativas.

Las competencias que esta asignatura aporta a los planes de estudio son las siguientes:

1. Desarrollo de habilidades para actualizar sus conocimientos y emprender estudios posteriores, incluyendo la especialización farmacéutica, la investigación científica y el desarrollo tecnológico, y la docencia.

2. Capacidad para recabar y transmitir información en lengua inglesa con un nivel de competencia similar al B1 del Consejo de Europa.

3. Módulo: Legislación y farmacia social. Dominar técnicas de recuperación de información relativas a fuentes de información primarias y secundarias (incluyendo bases de datos con el uso de ordenador) e informatizadas.

4. Módulo: Legislación y farmacia social. Conocer las técnicas de comunicación oral y escrita adquiriendo habilidades que permiten informar a los usuarios de los establecimientos farmacéuticos en términos inteligibles y adecuados a los diversos niveles culturales y entornos sociales.

A raíz de la construcción de nuestros proyectos docentes de la oposición a Profesores Titulares de Universidad (Ferragud, Lucas y Vidal) basados en la asignatura mencionada como memoria, tomamos conciencia de la necesidad de realizar los cambios adecuados para mejorar la situación de enseñanzaaprendizaje. De hecho, a través de tres proyectos de innovación consecutivos (Tabla 1) se ha reformulado tanto el contenido como la metodología docente de la asignatura. 


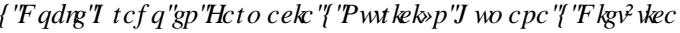

\begin{tabular}{|c|l|c|}
\hline CURSO & \multicolumn{1}{|c|}{ TÍTULO PROYECTO } & CÓDIGO \\
\hline $2018-2019$ & $\begin{array}{l}\text { Adaptación de la asignatura Documentación y Metodología } \\
\text { Científica del Grado en Farmacia a una metodología docente } \\
\text { guiada por la construcción de un trabajo (DOCiMET) }\end{array}$ & UV-SFPIE-RMD18-189811 \\
\hline $2019-2020$ & $\begin{array}{l}\text { Evaluación del proyecto DOCiMET referente a la reestructuración } \\
\text { de la asignatura Documentación y Metodología Científica del }\end{array}$ & UV-SFPIE-PID19-1096517 \\
\hline $2020-2021$ & $\begin{array}{l}\text { Grado en Farmacia } \\
\text { Científica del Grado en Farmacia en tiempos de Covid-19 }\end{array}$ & UV-SFPIE_PID20-1355845 \\
\hline
\end{tabular}

Durante los cursos anteriores a 2018, y tal como constaba en la guía docente, el contenido de la asignatura estaba configurado por cuatro tipos de sesiones (Figura 1): teóricas (clases magistrales), seminarios (aplicación práctica de conceptos teóricos), prácticas de informática (búsquedas en bases de datos) y tutorías presenciales (construcción de un mapa conceptual sobre un tema teórico). La calificación de la evaluación resultaba de la nota del examen teórico (50\%), la elaboración de un cuaderno de prácticas y seminarios $(40 \%)$ y la evaluación del mapa conceptual (10\%).

\section{Clases teóricas ( 25 horas) (2 sesiones $55 \mathrm{~min}$ )}

1. Presentación. Necesidades y usos de la información en farmacia

2. Fuentes primarias de información científica. Clasificación

3. Búsquedas bibliográficas

4. Bases de datos para la recuperación de información científica

5. Citas, impacto y la gestión de la información

6. Introducción general al curso en Metodología

7. Los métodos de la ciencia (I)

8. Los métodos de la ciencia (II)

9. La Revolución científica

10. Experimentación con animales
11. La terminología científica (I)

12. La terminología científica (II)

13. Los ensayos clínicos (I)

14. Los ensayos clínicos (II)

15. Ciencia, medicina y tecnología

16. La farmacia como disciplina y profesión

17. La industria farmacéutica

18. La comunicación científica (I)

19. La comunicación científica (II)

20. Repaso general del curso. Dudas. Preparación del examen

\section{Seminarios (10 horas)}

S1: Recursos de la UV. Catálogo de las bibliotecas de la UV

- S2: El artículo científico

- \$3: Gestores bibliográficos: Zotero

- S4: La experimentación humana

- S5: La cultura del medicamento

\section{$\checkmark$ Prácticas de informática (5 horas)}

- Sesión 1: Búsquedas en bases de datos en Ciencias de la Salud

- Sesión 2: Búsquedas en bases de datos multidisciplinares

\section{Tutorías presenciales ( 2 horas)}

Sesión 1: Técnicas para elaborar mapas conceptuales.

Sesión 2: El alumno deberá presentar un borrador del mapa

conceptual que pretende realizar. Exposición y resolución de dudas surgidas durante el curso relacionados con los contenidos teóricos

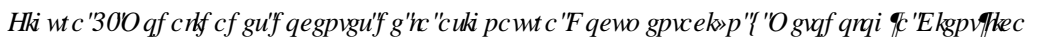

(cc) EY-NC-ND 2021, Universitat Politècnica de València

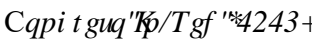


Partiendo de esta base hemos diseñado una estrategia docente que plantea la implicación del alumnado en una serie de actividades, previas, durante y después de las clases. Buscamos un aprendizaje significativo, que conecte con los intereses de los estudiantes, o al menos los provoque, y también con la realidad social. $\mathrm{Al}$ ser esta una asignatura que ayuda a buscar información científica de calidad, contribuye notablemente a desarrollar las competencias 1 y 3 .

En cuanto a la segunda competencia, hay que decir que impartimos en inglés íntegramente el grupo AR. Pero además, utilizamos textos y sugerimos lecturas que están en esta lengua. El alumnado debe familiarizarse necesariamente con el manejo del idioma actualmente más relevante en las publicaciones científicas.

Como se verá en los métodos que desarrollamos, el fomento del trabajo en parejas y en grupos contribuye a la implementación de las competencias 1 y 4. El intercambio de opiniones, la discusión científica, la producción de conocimiento y la comunicación de los resultados adecuadamente son estrategias necesarias en cualquier ámbito laboral al que se puede enfrentar en el futuro el alumnado de farmacia.

\section{Objetivos}

Objetivo 1. Implantar nuevas técnicas docentes utilizando las herramientas basadas en TIC, que mejoran la efectividad en la asimilación de los conceptos de la asignatura.

Objetivo 2. Facilitar la implicación del alumnado en su aprendizaje y autoevaluación en línea.

Objetivo 3. Fomentar el trabajo en grupo y la cooperación utilizando metodologías en línea como herramienta de mejora para el aprendizaje y valor añadido en el futuro laboral.

Objetivo 4. Mejorar la adquisición de las competencias de la asignatura, optimizando la estructura e hilo argumental de los contenidos con metodologías activas para la enseñanza y el aprendizaje.

Objetivo 5. Utilizar la situación sanitaria vivida como un elemento de reflexión sobre salud pública, investigación en biomedicina, vacunas y medicamentos, asistencia y ética.

Objetivo 6. Introducir al alumnado en la importancia de la interdisciplinaridad.

Objetivo 7. Formar al alumnado en valores adecuados desde una perspectiva ética para desarrollar su actividad profesional en una sociedad democrática.

Objetivo 8. Comprobar la satisfacción tanto de los y las estudiantes implicados como de los propios profesores y profesoras participantes en el proyecto.

Estos objetivos se adecuan a las siguientes líneas estratégicas de la UV: 1) Elaboración de material y diseño de estrategias para la docencia virtual y la evaluación en línea; 2) Diseño, organización, desarrollo y evaluación de la docencia; 3) Actualización de la docencia; 4) Metodologías activas para el aprendizaje; 5) Incorporación de los Objetivos de Desarrollo Sostenible (ODS) en la educación superior. 


\section{Desarrollo de la innovación}

A lo largo de los tres proyectos docentes mencionados se han desarrollado las siguientes innovaciones:

\section{Metodologías docentes}

- Métodos de enseñanza basados en las diferentes formas de exposiciones magistrales (Fernández, 2007).

- Métodos basados en el aprendizaje autónomo, mejorando los mecanismos de regulación del aprendizaje individual.

Se ha desarrollado la modalidad ) CSSHET \&CWURP para aumentar de forma significativa el grado de autonomía de los estudiantes. También se han diseñado materiales docentes innovadores y creado entornos de aprendizaje y de evaluación (Prieto, 2017; Bergmann, 2012).

- Métodos orientados a la discusión y trabajo en equipo. Se fomenta el aprendizaje colaborativo a través de la realización de un trabajo monográfico en grupos como eje vertebral de la asignatura. Se procura la distribución de roles, responsabilidades y tareas en el desarrollo de actividades colaborativas entre el alumnado de forma presencial o a través de los foros virtuales.

\section{Medios e instrumentos de evaluación}

Se han desarrollado mecanismos de mejora del aprendizaje en términos de autoevaluación y de evaluación continuada (Salas Perea, 2017; Caviscol, 2015; Universidad de Valencia, 2007).

- Evaluación de los contenidos adquiridos por el alumnado: Evaluación continua y Evaluación final.

- Evaluación del grado de satisfacción e implicación del alumnado: Cuestionarios.

- Autoevaluación del profesorado.

\section{Formación integral/competencial del alumno}

Como se ha podido comprobar a través de la lectura de las competencias propias de la asignatura que analizamos y la manera en que se enfoca, la formación que proponemos tiene un alcance, sin duda, integral. Desde nuestro punto de vista, un futuro titulado en una disciplina propia de las ciencias de la salud, que además aspira a convertirse en un profesional que desarrollará una labor de gran impacto sobre la calidad de vida de las personas, debe ser consciente de las enormes repercusiones de su actividad. Por ello nuestro planteamiento pretende ser lo más global posible, atendiendo a reflexiones que van más allá de las cuestiones técnicas, y que también plantea un acercamiento de carácter sociológico, antropológico y bioético. Pretendemos que nuestro estudiantado se plantee que la farmacia es una actividad que va más allá de un trabajo que desarrolla entre las paredes de un laboratorio.

\section{Nuevos escenarios formativos en educación superior}

A raíz de la implantación de la docencia híbrida durante el curso 2020-2021 ha sido necesario un replanteamiento de la metodología didáctica. Ello nos ha obligado a un uso de herramientas digitales y de redes sociales para la construcción y el intercambio de conocimiento.

\section{Incorporación de ODS en la educación superior}

La Facultad de Farmacia es centro piloto de la Universitat de València para la implantación de los ODS. En este sentido, la asignatura Documentación y Metodología Científica ha tenido una participación específica en la implantación de los ODS en nuestro alumnado de primer curso. Con ello hemos desarrollado una actividad dentro de uno de los temas que impartimos. 


\section{Recursos tecnológicos de apoyo al aprendizaje}

El principal valor de las innovaciones desarrolladas para la actualización de la asignatura reside en la vehiculización de los contenidos del temario independientemente del tipo de docencia teórica, práctica, seminarios, o tutorías grupales, mediante las técnicas más adecuadas dentro de un conjunto de recursos basados en TIC para la creación de una serie de actividades individuales y colaborativas (Rodríguez, 2009). Para el desarrollo de las sesiones teóricas, particularmente en actividades iniciales motivadoras, se han utilizado herramientas en línea como el Padlet o el Kahoot. Sin duda, ello estimula la participación y permite al o la docente ir desarrollando explicaciones que conectan con las respuestas que van dando los y las estudiantes. Para su conocimiento y uso los profesores han participado en diferentes cursos de formación de la UV.

Las herramientas que la propia aula virtual (Moodle) de la UV aporta al profesorado han servido para provocar la participación activa de los y las estudiantes. En este sentido, un buen ejemplo sería el desarrollo de foros previos a las sesiones teóricas.

La valoración de las actividades online a través del aula virtual resulta esencial como elemento empleado para la evaluación continua de la asignatura. Se ha trabajado con TIC de investigación y gestión de la información; TIC para el trabajo activo-colaborativo (organizar tutorías, reuniones y tareas); TIC de generación de mapas conceptuales, infografías y timelines que permiten crear nubes de palabras para trabajar el vocabulario de los temas, hacer brainstorming o analizar palabras de un texto; TIC para la creación de presentaciones originales, impactantes y motivadoras con contenido visual dinámico; y TIC para la evaluación, corrección, respuesta de audiencia y rúbricas (para hacer cuestionarios y crear actividades de participación en el aula).

\section{Resultados}

\section{Reformulación de la metodología docente de la asignatura a través del proyecto DOCiMET (UV-SFPIE-RMD18-189811)}

El desarrollo de este proyecto supuso la adaptación de la asignatura a una nueva metodología docente, donde el alumnado aplica los contenidos y las competencias adquiridas a la construcción de un trabajo monográfico que actuaba como eje central, tanto en la evolución de las sesiones como en la evaluación de la materia. El trabajo propuesto se realizó en grupos de hasta cuatro estudiantes, mediante herramientas y técnicas basadas en TIC, y tanto las sesiones teóricas como las prácticas y seminarios se impartieron mediante la modalidad ) CSSHEt \&QWURRP. El objetivo era conseguir que el estudiantado tomase conciencia de la importancia de la asignatura y consolidar los conocimientos más eficazmente gracias a la construcción del trabajo monográfico.

\subsection{Trabajo individual}

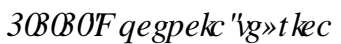

Siguiendo la línea de mejora de la asignatura se propusieron cuatro temas nuevos (Ciencias de la salud y género, La vida social de las medicinas, La construcción social de la enfermedad y Patentes farmacéuticas). Se elaboró una guía para los estudiantes y las correspondientes presentaciones en SRZHUSRIQMen las tres lenguas vehiculares.

- Modalidad ) CSSHCt\&DWRRP para el desarrollo de las sesiones teóricas que exige un trabajo continuo

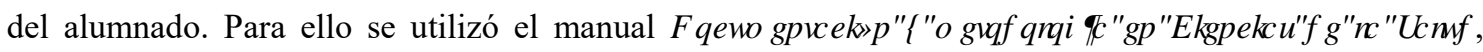
elaborado en 2017 por los profesores de la asignatura (Ferragud, 2017) (Figura 2), y otros materiales (textos, 
artículos, documentales, películas...) pensados y diseñados para la preparación de las clases teóricas y la reflexión personal, estimulando la participación del alumnado con metodologías diversas (resolución de dudas y planteamiento de retos a resolver en grupo y actividades con herramientas digitales), con la conducción y la parte expositiva del profesorado. Este texto se ha publicado también en versión digital para conseguir una mayor difusión.

- Cuestionarios referentes a la comprensión del tema expuesto (versión impresa y en línea a través del aula virtual)

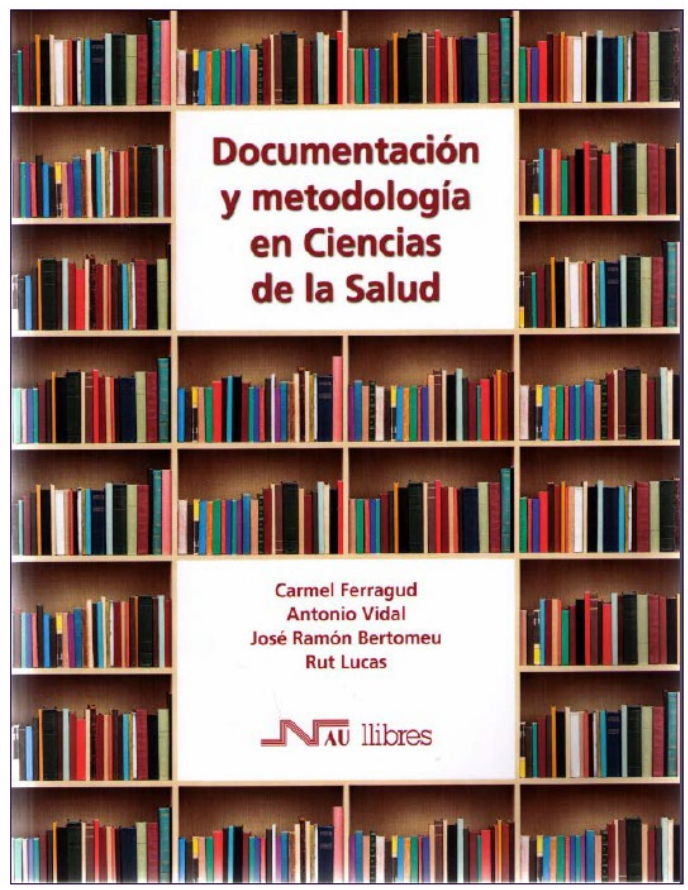

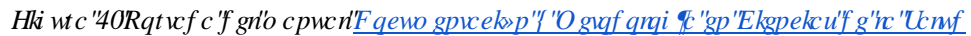

\section{पMUप' RFHQWDSU FWFD}

- Prácticas de informática para desarrollar las habilidades necesarias para realizar las búsquedas de información necesarias en el desarrollo del trabajo monográfico.

\subsection{Trabajo en equipo / colaborativo.}

\section{૧प⿴囗TWEDNRP RQRJU IIFR}

Se constituyeron equipos de trabajo de cuatro estudiantes a los que se les proporcionó un tema a desarrollar relacionado con los principales grupos farmacológicos. Con ellos debían aplicar los conocimientos obtenidos a partir de cada uno de los temas que constituyen el esqueleto de la asignatura. De este modo se pretendía conseguir la integración de todos los temas de documentación y metodología, a diferencia de los cursos anteriores en que toda la parte de documentación antecedía a la de metodología científica.

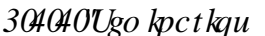

Los cinco seminarios de los que consta la asignatura se aprovecharon para que el profesorado pudiera conducir convenientemente las discusiones, ayudara en las decisiones que implicaba el trabajo grupal, observara los roles, la implicación y la participación. También para desarrollar búsquedas y gestión de la información científica con el fin de utilizar convenientemente las herramientas relativas a las tecnologías de la información y la comunicación. 


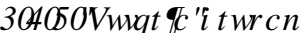

El objetivo principal era ofrecer una técnica de trabajo, el mapa conceptual, y acabar configurando un mapa referente al trabajo monográfico realizado, formando parte de la nota final.

\subsection{Evaluación de los contenidos}

La evaluación de la materia se realizó de la siguiente manera:

- Examen teórico $(60 \%)$

- Actividades prácticas $(20 \%)$

- Presentación del trabajo monográfico en grupo (20\%).

\section{Aplicación de las nuevas metodologías docentes DOCiMET en el curso 2019-2020. Evaluación de la innovación (UV-SFPIE-RMD18-189811)}

La implantación del proyecto DOCiMET la llevamos a cabo desde el convencimiento de que hay que adaptarse a los criterios didácticos más adecuados para estudiantes del siglo XXI, acercarse a los intereses y aportar contenidos y dinámicas de trabajo que resulten útiles y atractivas. A su vez, la evaluación del mismo es esencial para obtener información del estudiantado sobre la transformación que ha experimentado

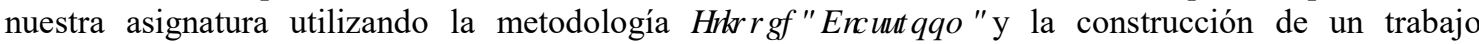
monográfico como eje central de los contenidos.

- Implantación de metodologías docentes basadas en ) CSSHEt\&CWMRRP y construcción de trabajo monográfico entre los meses de septiembre y diciembre de 2019.

- Creación de un grupo de discusión entre el profesorado y los representantes de alumnos de los grupos implicados (delegados y subdelegados). De esta manera, un colectivo de siete profesores, entre los que se encuentran tres licenciadas en farmacia, hemos reflexionado y trabajado muchas horas para conseguir modificar esta asignatura.

- Al término de la asignatura Documentación y Metodología Científica en diciembre del 2019, se realizó una reflexión conjunta entre profesores y estudiantes para evaluar su desarrollo a lo largo del cuatrimestre en cuanto a metodologías docentes, contenido y evaluación.

- Acta de la reunión mantenida. A raíz de la reunión mantenida con los delegados de todos los grupos para evaluar el funcionamiento de la asignatura con la metodología ) CSSHCE\&DWRRP pudimos evidenciar que había una mayor motivación y participación en los contenidos de la asignatura. Sin embargo, el alumnado manifestó que la dedicación que implicaba la nueva metodología no tenía un beneficio importante en la nota final ni formaba parte de un posible proceso de evaluación continua permitiendo la evaluación previa de contenidos (Tabla 2).

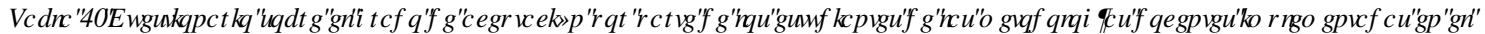

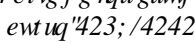

\begin{tabular}{|l|l|}
\hline & $\begin{array}{c}\text { OPINIÓN DEL ALUMNADO SOBRE LA ACTUALIZACIÓN DE LA METODOLOGÍA } \\
\text { DOCENTE (DOCiMET) }\end{array}$ \\
\hline FAVORABLE & $\begin{array}{l}\text { Integración global y equilibrada en los contenidos de la asignatura referentes al temario del } \\
\text { bloque de Documentación y del bloque de Metodología Científica }\end{array}$ \\
\hline FAVORABLE & $\begin{array}{l}\text { Sesiones teóricas de dos horas continuadas semanales resultan más eficientes al incorporar } \\
\text { actividades alternas en la modalidad ) CSSHEL\&CWRRP frente a la lección magistral }\end{array}$ \\
\hline
\end{tabular}




\begin{tabular}{|c|c|}
\hline FAVORABLE & $\begin{array}{l}\text { Verificación de mayor adquisición de conocimientos e integración de contenidos a través de } \\
\text { la construcción de un trabajo monográfico grupal }\end{array}$ \\
\hline FAVORABLE & $\begin{array}{l}\text { Se confirma la utilidad de la asignatura para el resto de asignaturas a lo largo del grado y en } \\
\text { el futuro profesional }\end{array}$ \\
\hline DESFAVORABLE & $\begin{array}{l}\text { Necesidad de adquirir el manual recomendado (edición impresa) para la asignatura en la } \\
\text { modalidad ) CSSHCE\&DWRRP. Hay ejemplares en la biblioteca pero son insuficientes. }\end{array}$ \\
\hline DESFAVORABLE & $\begin{array}{l}\text { Los alumnos deben revisar los textos recomendados por el profesorado antes de comenzar las } \\
\text { sesiones teóricas o prácticas en la metodología ) CSSHE\&QDWRRP }\end{array}$ \\
\hline DESFAVORABLE & $\begin{array}{l}\text { La necesidad de tomar apuntes durante las clases impide seguir correctamente las } \\
\text { explicaciones frente a la disponibilidad de diapositivas de la lección magistral }\end{array}$ \\
\hline DESFAVORABLE & $\begin{array}{l}\text { La modalidad ) ASSHG\&\&WRRP exige dedicación al alumnado que no se siente beneficiado } \\
\text { con una evaluación paralela, o posible eliminación de materia para el examen final }\end{array}$ \\
\hline DESFAVORABLE & $\begin{array}{l}\text { El esfuerzo realizado en la realización del trabajo monográfico no se ve compensado con un } \\
\text { beneficio en la eliminación de materia previa al examen final. }\end{array}$ \\
\hline DESFAVORABLE & $\begin{array}{l}\text { Contenido teórico y práctico extenso que habiéndose desarrollado a través de actividades que } \\
\text { requieren dedicación por parte de los alumnos no ha servido para eliminar materia }\end{array}$ \\
\hline
\end{tabular}

\section{Adaptación de la asignatura Documentación y Metodología Científica del Grado en Farmacia en tiempos de Covid-19 (UV-SFPIE_PID20-1355845)}

Atendiendo a las circunstancias especiales de semipresencialidad del curso académico 2020-2021 como consecuencia de la pandemia por COVID-19 (Almaiah, 2020; Watermeyer, 2020; Zuo, 2020), el tercer proyecto se planteó como una iniciativa orientada a la mejora de la calidad docente de la asignatura a través de iniciativas innovadoras enfocadas a, en primer lugar, hacer posible la adaptación de la docencia teórica y práctica a entornos virtuales o semipresenciales y, en segundo lugar, fomentar la evaluación continua del alumnado con la finalidad de provocar efectos positivos y constatables en el aprendizaje. La docencia semipresencial diseñada por el equipo de profesorado ha tenido como finalidad mejorar las desventajas notificadas por el alumnado durante el curso anterior así como permanecer en todo momento pendiente del alumnado como si de clases presenciales se tratara. Sin embargo, es evidente que la puesta en práctica de las metodologías desarrolladas en este proyecto implica un aumento en la independencia adquirida por los estudiantes recién llegados a la universidad. El resultado de las actividades que hemos desarrollado son los que exponemos a continuación.

\subsection{Docencia semipresencial}

Transformación general del estilo docente presencial de la asignatura a las condiciones de docencia semipresencial, que permite alternar entre modalidades síncronas y asíncronas, promoviendo un papel activo del estudiantado en los procesos y actividades de aprendizaje a lo largo de toda la asignatura. 


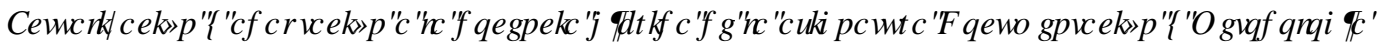 \&IHQWIFD $G H D^{*}$ WORIHQFarmacia!}

Además, hemos pretendido que la situación pandémica y todas las reflexiones que ha suscitado sean una oportunidad para la enseñanza de los futuros profesionales en las ciencias de la salud. Para facilitar el acceso a los materiales de consulta bibliográficos, se ha digitalizado el manual ' RFXP HQWFIy QI। [O HRRRRJtDHQ \&IHQFWVGH(D)6DXG que originalmente estaba en versión impresa, favoreciendo así su disponibilidad en formato en línea.

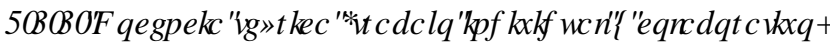

El contenido teórico de la asignatura, más relacionado con los temas de metodología científica, se ha impartido durante las sesiones teóricas establecidas en el horario.

En lo relativo a las sesiones de clases magistrales, y de acuerdo con la normativa de la Facultad de Farmacia de la UV, la docencia ha sido presencial hasta la primera semana de noviembre. No hay que olvidar que es el primer contacto de los alumnos de primer curso con la universidad. A partir de este momento, las clases teóricas han continuado mediante videoconferencia síncrona. Los materiales de cada tema se han colgado previamente en el aula virtual (diapositivas y lecturas de interés). Durante la sesión de trabajo en el aula, y luego con \%DFNERDCE\&RCDELWH se han realizado diversas estrategias participativas: foros de debate donde se invita a los estudiantes a participar con preguntas en torno a la sesión, cuestionarios, comentarios de fragmentos de vídeos, imágenes o artículos cortos. Las tareas realizadas por el alumnado se entregan a través del aula virtual y forman parte de la evaluación continua. Estas actividades se han alternado con los comentarios y explicaciones magistrales del profesorado apoyadas en presentaciones en SRZHUSRLQW

Los y las estudiantes han elaborado cinco ensayos a partir de los contenidos teóricos, donde plasmaron lo aprendido a través de las lecturas y trabajos previos realizados, las aportaciones de los compañeros en el foro y la reflexión personal. A modo de retroalimentación han recibido los comentarios individualizados del profesorado, y los ensayos muestran su evolución, reforzando así la idea de evaluación continua. Además se resolvieron dudas a través de tutorías individuales y grupales.

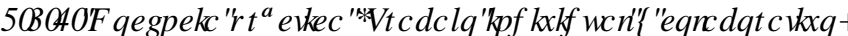

La teoría referida a documentación se ha integrado en el desarrollo de siete sesiones de carácter presencial (dos prácticas y cinco seminarios), desarrolladas por el alumnado a través de los ordenadores personales o dispositivos electrónicos. El acceso a los recursos electrónicos disponibles en la Biblioteca de la UV ha sido posible gracias a la conexión individual del alumnado a través de la Red Privada Virtual (VPN). Se ha recopilado cada ejercicio evaluable a través de la opción de "Tarea" del aula virtual de las actividades prácticas en el horario estipulado. Además, cada actividad lleva asociada un cuestionario en línea acerca del trabajo realizado, lo que permite la autoevaluación individual del alumnado a través de un sistema de retroalimentación y motivación continuada a lo largo del desarrollo de la asignatura durante todo el cuatrimestre.

Tutoría grupal. Los mapas conceptuales se comparten en el aula virtual favoreciendo la discusión del temario impartido a través de los foros.

\subsection{Evaluación continua}

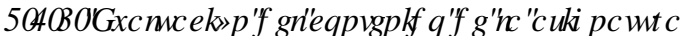

En el itinerario de evaluación continua el aprendizaje del alumnado se evaluó en torno a los dos tipos de modalidades docentes descritas:

- Evaluación por el profesor. Modalidad de evaluación que implica un proceso por el que el profesor mediante unos cuestionarios o ensayos y la realización de prácticas por parte del alumnado valorará los conocimientos adquiridos. 
- Autoevaluación del estudiantado. Modalidad de evaluación que implica un proceso mediante el cual el o la estudiante analiza y valora sus propias actividades realizando los cuestionarios en línea.

Las actividades evaluables y su participación en la nota fueron las siguientes: cinco ensayos referentes a la docencia teórica (40\%); dos prácticas de informática (20\%); cinco seminarios en aula de informática (20\%); mapas conceptuales $(10 \%)$; participación en foro $(10 \%)$

Respecto al itinerario con examen final, las actividades y su peso en la nota fueron: examen teórico (60\%); prácticas de informática y seminarios (40\%).

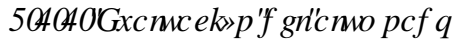

Evaluación en línea del grado de satisfacción e implicación del alumnado mediante la realización de un cuestionario anónimo a través del aula virtual (Figura 3, Tabla 3).

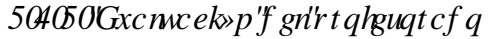

Autoevaluación a través de encuestas de satisfacción de la metodología empleada en términos de dedicación docente y resultados académicos del estudiantado; evaluación del profesorado a través de las encuestas a los alumnos realizadas por el Servicio de Formación Permanente e Innovación Educativa (6) 3, () de la UV. Cabe decir que la realización de este apartado está pendiente para el próximo mes de mayo, que es cuando se activan las encuestas de la Universidad de Valencia.

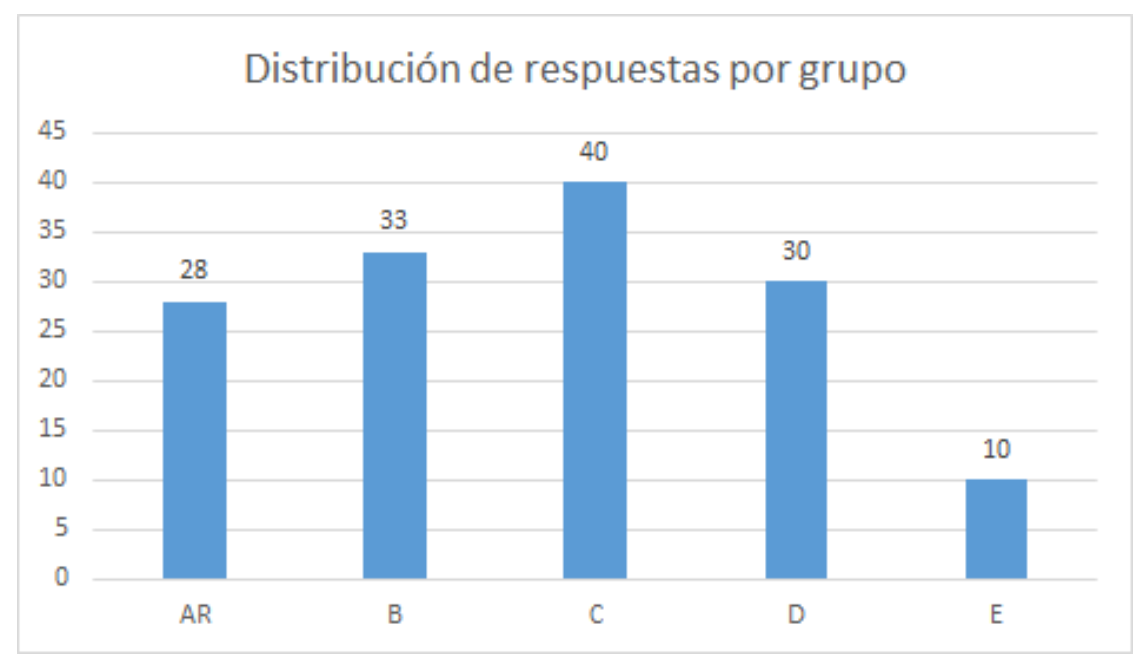

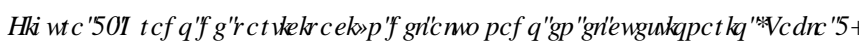




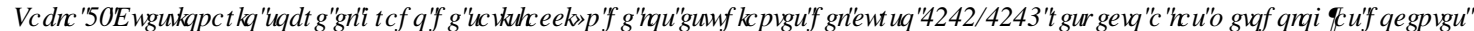
XULD] DODVHQGRFHQFDKKEUGD

\begin{tabular}{|c|c|c|c|c|}
\hline & $\begin{array}{l}\text { Muy de } \\
\text { acuerdo }\end{array}$ & De acuerdo & En desacuerdo & $\begin{array}{c}\text { Muyen } \\
\text { desacuerdo }\end{array}$ \\
\hline $\begin{array}{l}\text { Las clases teónicas de Metodología han sido } \\
\text { dinámicas y participativas desde el punto de vista del } \\
\text { planteamiento hecho por el profesorado }\end{array}$ & 39 & $85(60,3 \%)$ & 16 & 1 \\
\hline $\begin{array}{c}\text { Respecto al bloque de Metodología científica, el foro } \\
\text { me ha parecido que planteaba actividades } \\
\text { interesantes y varias }\end{array}$ & 38 & $90(63,8 \%)$ & 13 & 0 \\
\hline $\begin{array}{l}\text { Mi capacidad de escribir textos con cierta } \\
\text { complejidad ha mejorado }\end{array}$ & 33 & $79(56 \%)$ & 26 & 3 \\
\hline $\begin{array}{l}\text { Considero que ha sido una buena idea hacer las } \\
\text { sesiones de documentación de manera individual } \\
\text { con los ordenadores personales en aula de teoria }\end{array}$ & 58 & $66(46,8 \%)$ & 14 & 3 \\
\hline $\begin{array}{c}\text { Considero una metodología eficiente el hecho de } \\
\text { impartir el contenido de Documentación explicando } \\
\text { los conceptos teóricos de manera paralela con las } \\
\text { sesiones de prácticas y seminarios }\end{array}$ & 54 & $75(53,2 \%)$ & 11 & 1 \\
\hline $\begin{array}{l}\text { Las prácticas y seminarios de Documentación } \\
\text { considero que serán de mucha utilidad en el futuro }\end{array}$ & 46 & $79(56 \%)$ & 14 & 2 \\
\hline $\begin{array}{c}\text { Considero que la carga de trabajo de la evaluación } \\
\text { continua está compensada con no tener que hacer el } \\
\text { examen }\end{array}$ & $63(44,7 \%)$ & 60 & 18 & 0 \\
\hline $\begin{array}{c}\text { Si empezara ahora de nuevo la asignatura } \\
\text { renunciaría a la evaluación continua y optaría por el } \\
\text { examen }\end{array}$ & 2 & 2 & 29 & $108(76,6 \%)$ \\
\hline $\begin{array}{c}\text { Mi implicación general con la asignatura ha sido la } \\
\text { adecuada para lo que considero que tiene que hacer } \\
\text { un estudiante universitario }\end{array}$ & 47 & $84(59,6 \%)$ & 9 & 1 \\
\hline $\begin{array}{c}\text { La asignatura Documentación y Metodol ogía } \\
\text { científica me ha servido para tener una visión más } \\
\text { global y crítica de la ciencia, la medicina y la } \\
\text { farmadia. }\end{array}$ & $73(51,8 \%)$ & 65 & 3 & 0 \\
\hline & el foro & los ensayos & las clases teóricas & $\begin{array}{c}\text { la combi nadión de } \\
\text { las tres } \\
\text { modalidades } \\
\text { docentes }\end{array}$ \\
\hline $\begin{array}{l}\text { Con lo que más he aprendido de Metodología } \\
\text { científica ha sido con: }\end{array}$ & 26 & 37 & 22 & $56(39,7 \%)$ \\
\hline & \begin{tabular}{|c|} 
Sí, el manual \\
en soporte \\
papel a través \\
de la \\
biblioteca \\
\end{tabular} & $\begin{array}{c}\text { Sí, he adquirido } \\
\text { la versión online } \\
\text { del manual }\end{array}$ & $\begin{array}{c}\text { Si, he adquirido la } \\
\text { versión en } \\
\text { soporte papel del } \\
\text { manual }\end{array}$ & $\begin{array}{l}\text { No lo he } \\
\text { consultado }\end{array}$ \\
\hline $\begin{array}{l}\text { ¿Has consul tado el manual de Documentación y } \\
\text { Metodología dientífica? }\end{array}$ & $40(28,4 \%)$ & $40(28,4 \%)$ & 28 & 33 \\
\hline
\end{tabular}




\subsection{Incorporación de los ODS en la educación superior}

Se desarrolló la actividad diseñada por la Cátedra de Cooperación y Desarrollo Sostenible de la UV, "Curso de iniciación a los ODS. ¡Sé parte del cambio de todas las personas!”. Se realizaron seis actividades que incluían videos y cuestionarios a través del aula virtual con el objetivo de fomentar el conocimiento de los ODS (Figura 4).

CURSO DE INICIACIÓN A LOS ODS. ¡SÉ PARTE DEL CAMBIO DE LAS PERSONAS!

\section{Curso de iniciación a los ODS. ¡Sé parte del cambio de TODAS las personas!}

Wi Web Cátedra de Cooperación y Desarrollo Sostenible
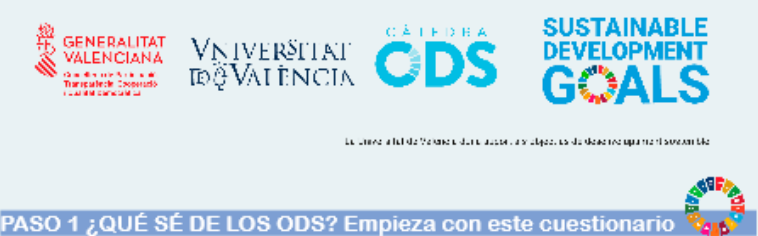

[Q] ¿Qué sabes de los ODS?
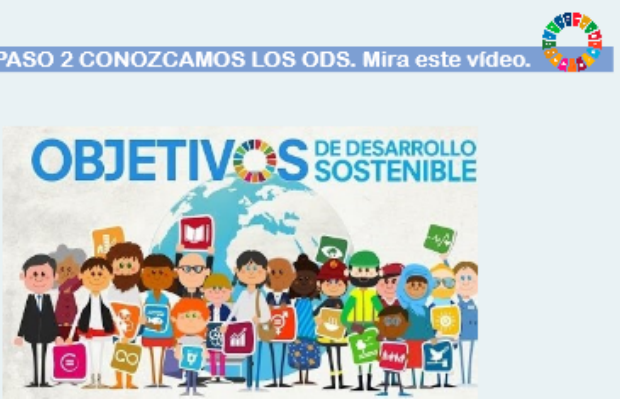

Figura 4. Curso de iniciación a los ODS. ¡Sé parte del cambio de todas las personas!. Cátedra de Cooperación y Desarrollo Sostenible. Universitat de València.

\subsection{Actividades de difusión y transferencia del conocimiento}

Participación en el VII Encuentro de estudiantes de primer curso del Grado en Farmacia y del Doble Grado en Farmacia y Nutrición Humana y Dietética de la UV con la ponencia: "Farmacéuticos en un mundo globalizado: informados, críticos y comprometidos". Valencia, 4 de Noviembre de 2019.

Participación en la XVII Jornada sobre la Història de la Ciència i Ensenyament "Antoni Quintana Marí", organizada por la Societat Catalana d'Història de la Ciència i de la Tècnica (IEC) con la ponencia: "La història com a ferramenta per a entendre la ciència farmacèutica del present". Barcelona, 15 y 16 de noviembre de 2019. 


\section{Conclusiones}

La inquietud del profesorado por mejorar esta asignatura ha sido permanente con el desarrollo de tres proyectos de innovación docente durante tres cursos consecutivos. El principal resultado ha sido la transformación general del estilo docente en el desarrollo de la asignatura, fomentando la implicación constante del alumnado a lo largo de toda la asignatura a través de las diferentes metodologías descritas. A su vez, hemos pretendido acercar los temarios a los intereses y posibles conocimientos de los estudiantes. Hemos evaluado si los alumnos se han sentido atraídos y han conectado con los contenidos y actividades propuestas. También, como parte fundamental del engranaje, debemos autoevaluar nuestra labor como docentes, reflexionar sobre ella y continuar mejorándola para adaptarnos a las circunstancias cambiantes de la sociedad que nos rodea. Somos conscientes, y así nos lo han confirmado los alumnos de cursos superiores y egresados, que el resultado de los conocimientos adquiridos en la asignatura Documentación y Metodología Científica se aprecia mejor a lo largo del Grado y en la elaboración de los Trabajos de Fin de Grado (TFG), tanto en lo concerniente a los conocimientos adquiridos a la hora de acceder a recursos bibliográficos, así como en el desarrollo de un trabajo científico en el ámbito académico y posteriormente en cualquiera de los escenarios profesionales del farmacéutico donde resulta fundamental la capacidad de toma decisiones bien informadas en el contexto de la salud.

\section{Referencias}

Almaiah, M. A., Al-Khasawneh, A., y Althunibat, A. (2020). "Exploring the critical challenges and factors influencing the E-learning system usage during COVID-19 pandemic" en ( GXFDURQDQQG, QRRP DURQ7FFKQRRJIHN, 25, 5261-5280 <https://doi.org/10.1007/s10639-020-10219-y>.

ANECA (2004). /IELR EQDQRR GHD JWOR HQ ) DPDFID (ANECA, Ed.). Madrid: ANECA.

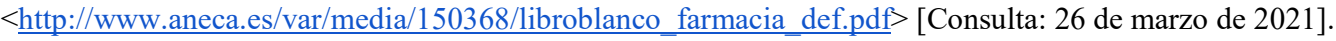

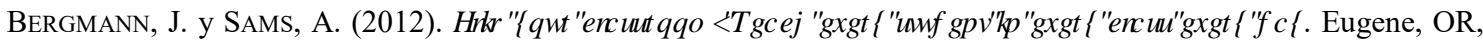
EE.UU.: International Society for Technology in Education.

CABISCOL, A. (2015). "La evaluación como reguladora del proceso de aprendizaje en la clase invertida" en ( VXXHD, $11-12$.

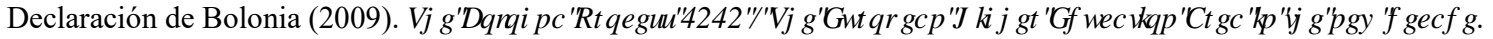
$<$ https://media.ehea.info/file/2009_Leuven_Louvain-la-Neuve/06/1/Leuven_Louvain-la-

Neuve_Communique_April_2009_595061.pdf $>$ [Consulta: 26 de marzo de $\overline{2}$ 2021].

FERNÁNDEZ, M. L. (2007). "Planificación del aprendizaje", en Fonseca M. C. y Aguaded J. I. ( QMKDUHQ (D)

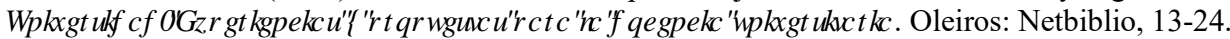

Ferragud, C., Vidal-Infer, A., Bertomeu, J. R. y LuCAS, R. (2017). ' RFXP HQWFIyQI $\square$ PHRQRQJTDHQ\&IHQFIDVGH (D)6DXG Valencia: Nau Llibres.

$<$ https://books.google.es/books?id=EV81DwAAQBAJ\&printsec=frontcover\&hl=ca\&source=gbs_ge summary_r\&ca

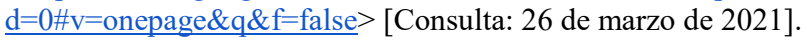

PRIETO, A (2017). Flipped classroom ¿Cuáles son sus ventajas? ¿Cuál es su origen y su evolución posterior? ¿Por qué no es una moda más? ¿Por qué mejora el aprendizaje? ¿Por qué deberías leer sobre este modelo en este verano? [Sitio web]. < http://profesor3punto0.blogspot.com.es/2016/07/flipped-classroom-cuales-son-sus_7.html> [Consulta: 26 de marzo de 2021].

Rodríguez, G., Ibarra, M., Dodero, J., Gallego, B., y CABeza, D. (2009). "Developing the e-Learning-oriented e-

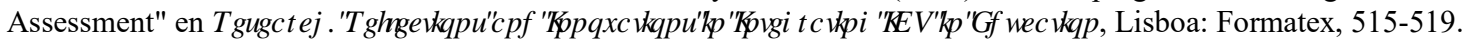

Salas Perea, R. S. y Salas Mainegra, A. (2017). "Evaluación para el aprendizaje en ciencias de la salud" en ( GXP HFHQR, 9, 1, 208-227. 


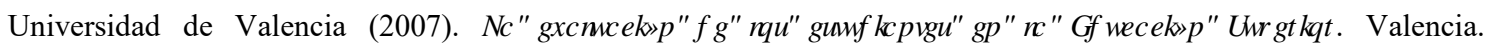
$<$ http://www3.uji.es/ betoret/Formacion/Evaluacion/Documentacion/La evaluacion estudiantes en la ESuperior UV.pdf $>$ [Consulta: 26 de marzo de 2021].

WAtermeyer, R., Crick, T., KNight, C. y Goodall, J. (2020). "COVID-19 and digital disruption in UK universities: afflictions and affordances of emergency online migration" en +LJKHU (GXFDMRQ 81, 623-641 $<$ https://doi.org/10.1007/s10734-020-00561-y>.

Zuo, L., Dillman, D., \& Miller Juve, A. (2020). "Learning At-Home During COVID-19: A Multi-institutional Virtual Learning Collaboration" en 0 HAFDO (GXFDMRQ 54, 7, 664-665 <https://doi.org/10.1111/medu.14194> [Consulta: 26 de marzo de 2021]. 